\title{
A CMS Luminosity Monitor Using Single-Crystal CVD Diamond Pixel Detectors
}

\author{
E. Bartz, ${ }^{a}$ J. Doroshenko, ${ }^{b}$ V. Halyo, ${ }^{b}$ B. Harrop, ${ }^{b}$ D.A. Hits, ${ }^{a}$ A. Macpherson, ${ }^{c}$ \\ D. Marlow, ${ }^{b}$ L. Perera, ${ }^{a, 1}$ S. Schnetzer ${ }^{a}$ and R. Stone ${ }^{a, 2}$
}

\author{
${ }^{a}$ Department of Physics and Astronomy, Rutgers University, \\ Piscataway, NJ 08854, U.S.A. \\ ${ }^{b}$ Department of Physics, Princeton University, \\ Princeton, NJ 08542, U.S.A. \\ ${ }^{c} C E R N$, \\ CH 1211, Geneva 23, Switzerland \\ E-mail: Stone@physics.rutgers.edu
}

\begin{abstract}
The Pixel Luminosity Telescope (PLT) is an innovative luminosity monitor which is planned as an upgrade for the CMS detector at the LHC. It uses pixelated single-crystal diamonds as the active sensor material bumpbonded to standard CMS pixel readout electronics. The PLT makes use of a presently unused feature of the pixel readout chip which was designed to provide a hit-over-threshold signal to a hardware trigger processor. This feature will produce a bunch crossing ( $25 \mathrm{~ns}$ ) 'hit' signal that can form the basis of luminosity information at the hardware level. We will report on the first successful use of this 'Fast-Or' signal to self-trigger on beta particles from a source using a diamond pixel detector. Expected performance of the PLT based on Pythia simulations is also detailed.
\end{abstract}

KEYWORDS: Particle tracking detectors, Large detector systems for particle and astroparticle physics

\footnotetext{
${ }^{1}$ Current address: University of Iowa

${ }^{2}$ Corresponding author.
} 


\section{Contents}

1 Introduction 1

2 PLT design 1

3 Production plane testing technique 4

4 Performance 4

4.1 Signal rates 5

4.2 Accidental rates 5

4.3 Track overlaps 5

4.4 Acceptance uniformity 5

4.5 Collision point centroid 5

5 Summary 6

\section{Introduction}

The proposed Pixel Luminosity Telescope (PLT) will provide bunch-by-bunch luminosity measurements. It will include additional features, such as determining any movement of the centroid of the collision point. The PLT is a dedicated luminosity monitor that will be independent of the CMS trigger and other detector components. In comparison to other CMS sub-detectors, the PLT is a relatively simple device that can provide stable performance over the lifetime of the LHC. It will provide precise bunch-by-bunch luminosity measurements with a statistical precision of $1 \%$, achievable within a few seconds. It can be calibrated to measure absolute luminosity using well know Standard Model processes. The PLT will have small systematic errors (below 1\%) and have a linear response over the full LHC luminosity range. Using a secondary readout mode it will be self-monitoring and self-calibrating by measuring its efficiency and background levels.

\section{PLT design}

The PLT consists of two sets of eight telescopes as shown in figure 1, each situated on either side of the collision point at $r \sim 5 \mathrm{~cm}$ and $z \sim 175 \mathrm{~cm}$. Each telescope will consist of three equally spaced sensor planes with a total length of $7.5 \mathrm{~cm}$. The active element of each telescope plane will be a $4 \mathrm{~mm} \times 4 \mathrm{~mm}$ single-crystal diamond sensor, covered with a 1,040 pixel array with a pixel pitch of $100 \mu \mathrm{m} \times 150 \mu \mathrm{m}$. Each diamond sensor will be bump-bonded to a standard CMS pixel readout chip (PSI46v2 ref. [2]).

Diamond sensors are used for this application because of their inherent radiation hardness, even when used at or above room temperature. This is critical for the PLT because there is simple 


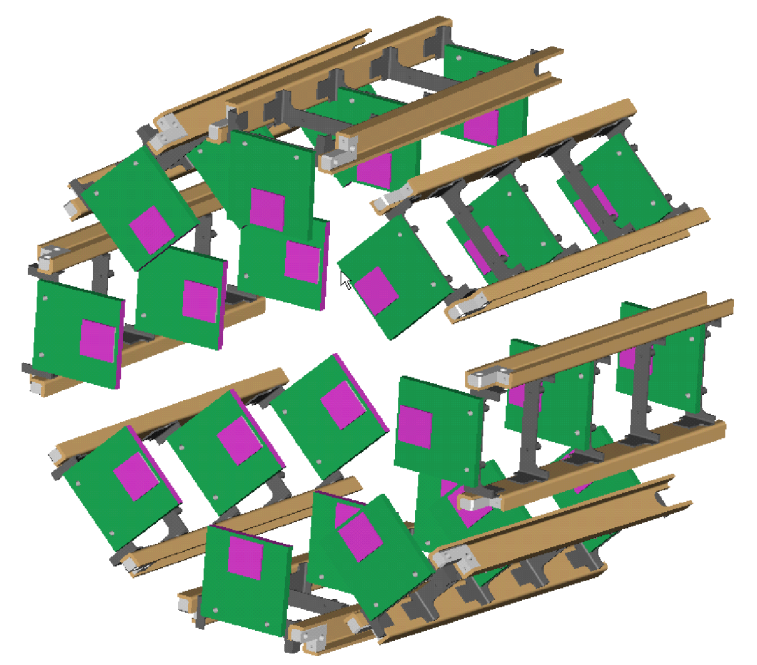

Figure 1. Design drawing of one half of the PLT. It consists of 8 telescopes with 3 planes, focused at a 1 degree angle onto the interaction point. The sensors are located at $\mathrm{r} \sim 5 \mathrm{~cm}$ and $\mathrm{z} \sim 175 \mathrm{~cm}$.

no space to provide the cooling necessary for radiation hard silicon detectors. The PLT sensors will need to survive more than $10^{15}$ protons $/ \mathrm{cm}^{2}$ over the lifetime of the LHC. Measurements of the radiation hardness of diamond show that it will survive this fluence with little loss of signal ${ }^{3}$. Since diamond is an inherently good electrical insulator, the diamond sensor leakage current is very low $(<10 \mathrm{pA})$, contributing a negligible amount to the frontend electronic noise. After exposure to radiation the leakage current becomes even lower.

Chemical Vapor Deposited (CVD) diamond can be manufactured into two distinct forms for use in charged particle detectors: single-crystal and polycrystalline. We have chosen to use singlecrystal diamond because of the significantly larger separation of the pulse height distribution from zero signal as show in figure 2. For a typical pixel threshold setting of $\sim 3,000$ electrons, this ensures the stability of hit efficiency necessary for low systematic errors.

The PLT will employ two readout modes for obtaining data from the diamond pixel sensors. One mode will use a feature of the CMS pixel readout chip (ROC) which can supply a fast signal (Fast-Or) for any bunch crossing which has one or more pixels with a pulse height above threshold. The Fast-Or signal from each of three planes will form a three-fold coincidence for each telescope in every ( $25 \mathrm{~ns}$ ) bunch crossing, which then forms the basis of the luminosity measurement. The second readout mode will be less frequent ( 1 to $10 \mathrm{KHz}$ ) but contain the full pixel readout information (pixel addresses and pulse heights). The full pixel readout mode will provide information for track reconstruction and will be used for diagnosis and corrections for the Fast-Or readout mode in terms of accidentals and track overlaps. Full pixel mode can also be used for determination of the collision point centroid as well as beam halo content. A block diagram of the data acquisition for the PLT is show in figure 3.

The PLT uses much of the standard CMS pixel hardware and software infrastructure. This includes a complex VME board to readout the optically encoded analog signals from the ROC called the Front End Digitizer (FED). The full pixel readout mode is performed using such a pixel FED. 


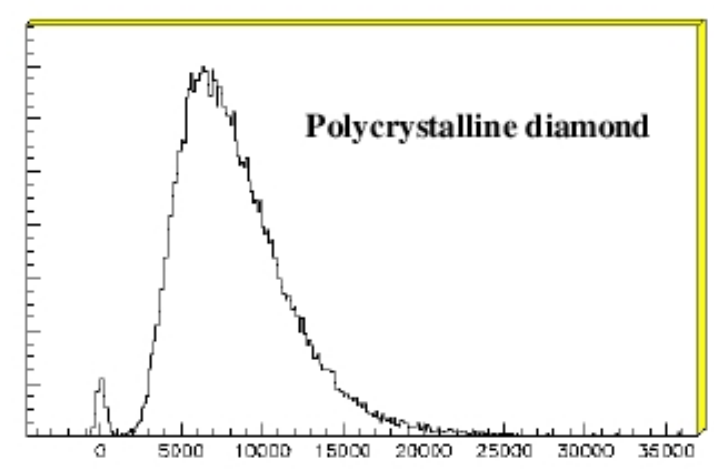

Signal (e-)

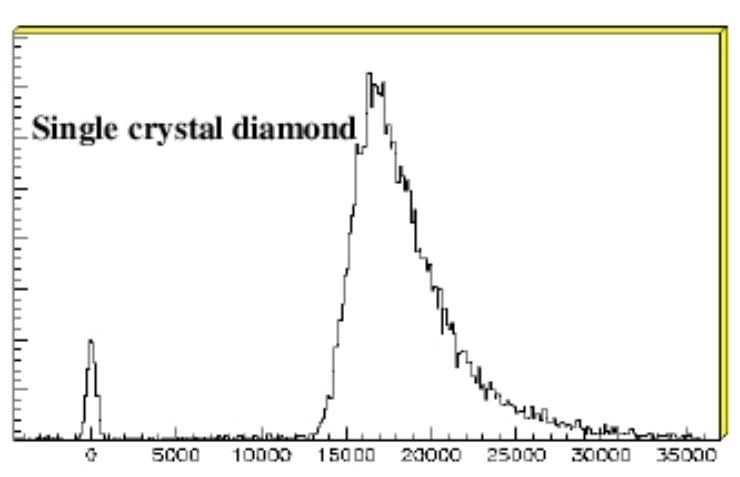

Signal (e-)

Figure 2. A comparison of minimum-ionizing pulse height distributionsg for $500 \mu \mathrm{m}$ thick polycrystalline diamond (left) and single crystal diamond (right) detectors. The narrow distributions centered at zero electrons are a sampling of pedestal events to show system noise $(\sim 300 \mathrm{ENC}$ for the measurement system employed).

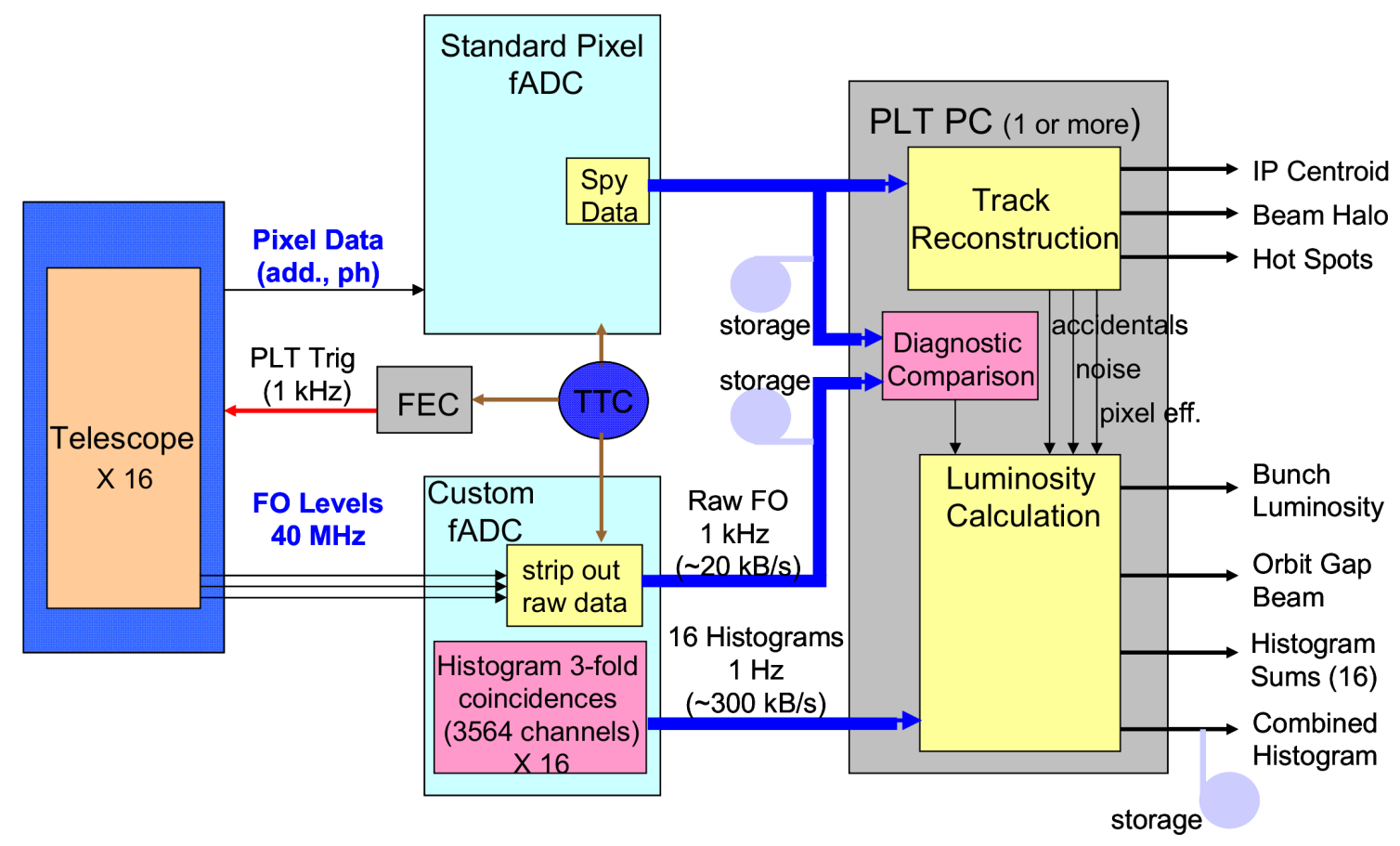

Figure 3. Data flow diagram for the PLT detector.

Also using the same FED hardware, but having customized firmware for the PLT, is a "Custom FED" for readout of the Fast-Or signals. The Custom FED produces a set of 16 histograms (one for each telescope) in which each bin corresponds to one of the 3,564 bunches within an LHC orbit. Data from both the Fast-Or FED and full pixel readout mode FED will be sent to the PLT DAQ computer. There the full readout pixel data will be used to provide corrections to the Fast-Or luminosity for effects due to accidental hits, noisy and inefficient pixels. It will also calculate the collision point 

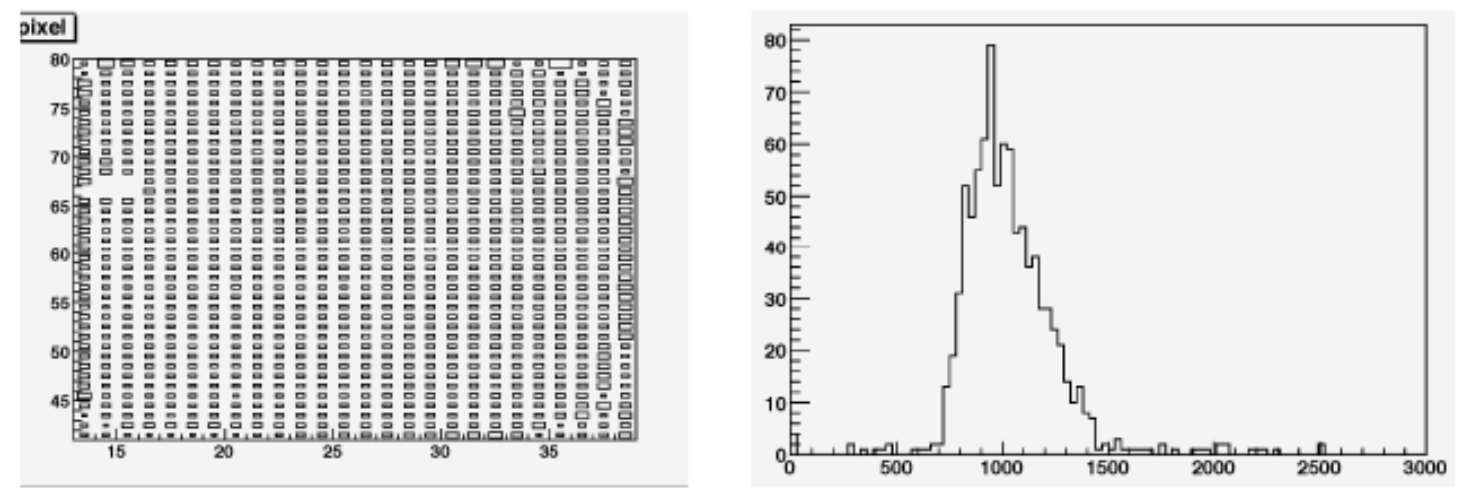

Figure 4. Pixel occupancy from self-triggering on ${ }^{90} \mathrm{Sr}$ electrons. The left plot shows pixel column vs. row relative occupancy by the size of the pixel rectangle. The large box in the upper right is probably a noisy pixel. Empty boxes are pixels either turned off (due to excess noise) or having disconnected bumpbonds. The plot on the right shows the number of pixels vs. number of hits per pixel.

centroid, the amount of beam halo, and the location of potential hot spots within the CMS detector. The corrected luminosity histograms will be delivered to CMS where they will be used in physics analyses and to the LHC control room where they can be used for monitoring beam conditions.

\section{Production plane testing technique}

To date, six prototype telescope planes have been built and tested on the bench. Some of these will be taken to a testbeam where detailed performance measurements will be made to determine hit efficiency, spatial resolution and signal uniformity. Additionally, irradiated detectors will be tested to ensure that the luminosity measurement capability is not compromised by radiation effects.

Taking all prototype or production planes to a testbeam is not feasible. Therefore, we have developed a quick diagnostic readout mode to determine how well a given telescope plane performs. This test mode uses a Strontium- 90 beta source to uniformly illuminate the sensor area. The trigger for reading out the sensor data relies solely on the Fast-Or signal from the ROC and is fed back into the pixel controller logic to generate, with suitable latency, a full pixel mode trigger signal to the ROC. Source betas that are self-triggered in this way are largely not minimum ionizing. The observable distribution of ionization charge starts at the pixel threshold level and extends to many times minimum ionizing. Nevertheless, the rate of pixel events is quite large for a typical laboratory source (hundreds of $\mathrm{Hz}$ ) and one can use this technique to quickly find the relative pixel-to-pixel efficiency and spot dead or noisy pixels easily. Figure 4 shows a pixel occupancy map for a prototype PLT detector plane as well as the distribution of hits per pixel for this device showing less than $1 \%$ out of $\sim 1,000$ pixels which are dead or have abnormally low hit rates.

\section{Performance}

The performance of the PLT with respect to signal rates, accidental rates, track overlaps, acceptance uniformity and precision of the location of the collision point centroid have been evaluated using Pythia and the official CMS geometry file. These results are summarized below. 


\subsection{Signal rates}

The number of tracks per pp interaction is found to be 0.005 per telescope. This number is only an estimate since Pythia multiplicities and rapidity distributions are not, of course, tuned to the LHC data but the uncertainty should be less than a factor of two. Assuming 21 interactions per bunch crossing at full luminosity, $\mathrm{L}=10^{34} \mathrm{~cm}^{-2} \mathrm{~s}^{-1}$, there will be on average 1.6 tracks in the PLT per bunch crossing. With an orbit gap period of $88 \mu \mathrm{s}$, this allows the relative luminosity of each bunch to be determined with a statistical precision of $1 \%$ in $0.55 \mathrm{~s}$.

\subsection{Accidental rates}

The percentage of the 3-fold coincidences due to accidental hits as a function of luminosity was studied. The accidental fraction is $1 \%$ at a luminosity of $10^{33} \mathrm{~cm}^{-2} \mathrm{~s}^{-1}$ and increases to about $4 \%$ for $\mathrm{L}=10^{34} \mathrm{~cm}^{-2} \mathrm{~s}^{-1}$. During operation, the accidental fraction can be readily determined using the tracking information available from the full pixel mode readout allowing it to be corrected to a small fraction of itself. As a result, systematic uncertainties due to accidentals should be below $1 \%$.

\subsection{Track overlaps}

A source of nonlinearity of the rate of 3 -fold coincidence with luminosity is due to the possible overlap of two or more tracks on a single detector plane. This will cause an underestimate of the number of tracks, called "hit robbing." The hit robbing fraction is $1.7 \%$ at a luminosity of $10^{33} \mathrm{~cm}^{-2} \mathrm{~s}^{-1}$ and increase to about $7 \%$ at $\mathrm{L}=10^{34} \mathrm{~cm}^{-2} \mathrm{~s}^{-1}$. As in the case of accidentals, the tracking information obtained from the full pixel readout mode will allow the hit robbing fraction to be reduced to below $1 \%$. The hit robbing effect can be further reduced by making use of the analog nature of the Fast-Or signals. The signal is an analog level that corresponds to the number of double columns hit in each detector plane in each bunch crossing. Using this feature, the area of the track overlap confusion can be reduced to the area of one double column, $300 \mathrm{um} \times 4 \mathrm{~mm}$, which reduces the hit robbing rate to $1.7 \%$ at $\mathrm{L}=10^{34} \mathrm{~cm}^{-2} \mathrm{~s}^{-1}$.

\subsection{Acceptance uniformity}

Because of the possibility of either short-term fluctuations or long-term drifts of the location of the collision point, it is important for the PLT to have an acceptance that is flat to better than $1 \%$ for detection of particles originating anywhere in the range of possible collision point locations. This has been achieved in the symmetry of the PLT design. The acceptance determined from Pythia shows that it is flat out to $+/-320 \mathrm{~mm}$ for drifts of the collision point along the $\mathrm{z}$-axis and is flat out to $12 \mathrm{~mm}$ for drifts in the collision point in the radial direction toward one of the telescopes. Even in the worst case, when the collision point drifts in the radial direction between two telescopes, the acceptance remains flat to $1 \%$ out to $4 \mathrm{~mm}$ radial drift (see figure 5).

\subsection{Collision point centroid}

Readout of the full pixel data from the PLT, in addition to providing corrections to the systematic uncertainties of the Fast-Or luminosity information, will allow the collision point centroid to be determined to a high precision. Using the location of the pixel hits, tracks can be extrapolated back to their origin. Due to the small angle of the telescopes with respect to the beam axis and the 

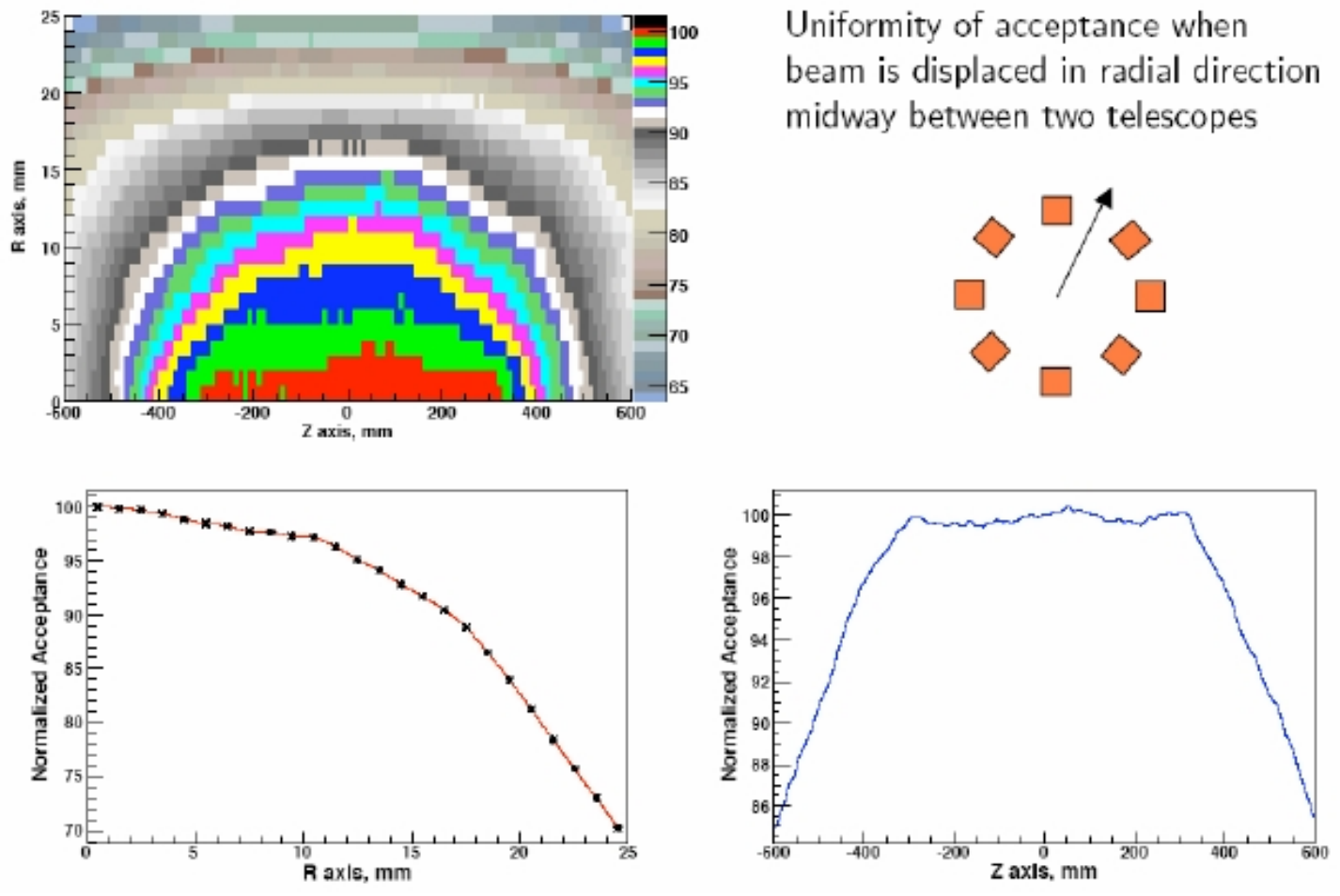

Figure 5. The acceptance is show above in a simulation where the beam moves in a (worst case) direction between two planes. The plot on the left shows the two dimensional acceptance in $\mathrm{r}$ and $\mathrm{z}$ where the color (right axis label) denotes the percentage acceptance. The bottom two plots are the one dimensional projections in $\mathrm{r}$ and $\mathrm{z}$. In $\mathrm{r}$, the acceptance is flat to $1 \%$ out to a $4 \mathrm{~mm}$ beam drift. Along the beam line $(\mathrm{z})$ the acceptance is flat to $+/-300 \mathrm{~mm}$.

short length of the PLT, it will not be possible to determine the curvature of the tracks. Taking into account the expected bunch length in $\mathrm{z}$ which will further smear out the interaction point, the PLT can nevertheless make a precise determination of the IP centroid. Assuming only digital pixel hit resolution in a plane, the PLT can determine the IP centroid to a precision of $12 \mu \mathrm{m}$ radially and $200 \mu \mathrm{m}$ in $\mathrm{z}$ in every luminosity data-section (93 seconds long).

\section{Summary}

The PLT is a dedicated luminosity monitor for CMS that is planned to be an early CMS detector upgrade. If approved by CMS, built and installed, it will provide precision measurements of the relative bunch-by-bunch luminosity, beam halo, beam hot spots, abort gap particles, and the relative collision point centroid location in real time (a few seconds). It will be a simple system with few data channels that will provide a standard and stable luminosity reference throughout the lifetime of CMS. Due to its capability to provide full pixel readout information, it will be self-monitoring and self-correcting for both efficiency and acceptance. Since it utilizes much of the electronics and software designed for the CMS pixel system, it can be built at a modest cost and effort. Additionally, it will provide a long term in-situ test of radiation-hard diamond pixel sensors under real experimental conditions. 


\section{References}

[1] C.G. Lester and D.J. Summers, Measuring masses of semi-invisibly decaying particle pairs produced at hadron colliders, Phys. Lett. B 463 (1999) 99 [hep-ph/9906349].

[2] M. Barbero et al., Design and test of the CMS pixel readout chip, Nucl. Instrum. Meth. A 517 (2004) 349.

[3] W. Adam et al., Radiation hard diamond sensors for future tracking applications, Nucl. Instrum. Meth. A 565 (2006) 278. 